\title{
Image analysis of TEM pictures of fluorine-intercalated graphite fibers
}

\author{
K. Oshida \\ Department of Electronics and Computer Science, Nagano National College of Technology, \\ Nagano 381, Japan \\ M. Endo \\ Department of Electrical Engineering, Faculty of Engineering, Shinshu University, Nagano 380, Japan \\ T. Nakajima \\ Division of Molecular Engineering, Faculty of Engineering, Kyoto University, Sakyo-ku, \\ Kyoto 606, Japan
}

S. L. di Vittorio

Department of Materials Science and Engineering, Massachusetts Institute of Technology, Cambridge, Massachusetts 02139

\author{
M. S. Dresselhaus \\ Department of Physics and Department of Electrical Engineering and Computer Science, Massachusetts \\ Institute of Technology, Cambridge, Massachusetts 02139 \\ G. Dresselhaus \\ Francis Bitter National Magnet Laboratory, Massachusetts Institute of Technology, Cambridge, \\ Massachusetts 02139
}

(Received 9 March 1992; accepted 23 October 1992)

\begin{abstract}
A digitization of the TEM pictures of fluorine-intercalated graphite fibers has been used to carry out quantitative measurements of the defect structure of this material. Emphasis is given to both the computer analysis technique and to the characterization of the defects. The amount of intercalation-induced disorder increases with increasing fluorine concentration. The fast Fourier transform of the digitized TEM image exhibits two diffuse spots, corresponding to the $c$-axis repeat distance of the intercalation compound. The length and width of the spots are a measure of the out-of-plane and in-plane disorder present in the fibers. From the fast Fourier transform, the distribution of interlayer repeat distances and the fraction of unintercalated graphite regions throughout the material is obtained. By selecting a small range of repeat distances and carrying out an inverse fast Fourier transform, the spatial distribution of material with a given repeat distance is determined. Regions with the same repeat distance are found to form islands. This particular feature of fluorine graphite intercalation compounds, as well as the nature of the microscopic defects and the staging behavior of fluorine-intercalated graphite fibers, are discussed in connection with the dual covalent and ionic nature of the carbon-fluorine bond in fluorine-intercalated graphite.
\end{abstract}

\section{INTRODUCTION}

Many characterization techniques are used to measure quantitatively the amount of disorder present in a material. Among the most widely used in the field of carbon-based materials are transport, Raman scattering, and $\mathrm{x}$-ray diffraction. ${ }^{1,2}$ By measuring such parameters as the resistivity and its temperature dependence, the magnitude and size of the magnetoresistance, and the magnitude of the disorder-induced peak in the Raman spectrum, it is possible to obtain a reliable numerical estimate of the magnitude of the disorder in the fibers (specified by the sheet resistance $R_{\square}$, the Raman linewidth, and crystallite sizes in the $a$ and $c$ directions
$L_{a}$ and $L_{c}$ ). However, these techniques do not provide much information on the specific nature of the disorder present in the material. Furthermore, such measurements yield information about the disorder, averaged over the whole sample investigated, whereas many disordered solids are found to be inhomogeneous.

Transmission Electron Microscopy (TEM) is a complementary technique for analyzing disordered materials, since it gives highly detailed information on the nature of the disorder in a very small sample of a solid. TEM is now a highly developed technique for structural characterization. ${ }^{3-5}$ Lattice fringe images have been used successfully with $100-400 \mathrm{kV}$ TEM instruments (the 
work reported here is mainly at $300 \mathrm{kV}$ ) to characterize well-ordered materials, such as ionic Graphite Intercalation Compounds (GIC's) where the superlattice structure can be easily seen and defects associated with point defect, dislocations, and crystallite boundaries can be identified. ${ }^{6}$ Unfortunately, the nature of the information obtained from TEM pictures was until recently mainly qualitative, and characterizing the defect structure by TEM becomes increasingly difficult as the amount of disorder is increased.

In this paper, we show how digitization of a high quality TEM image and subsequent computer analysis of the digitized picture can be used to obtain quantitative measurements of the nature and amount of disorder in a sample that exhibits a substantial amount of disorder. Fluorine-intercalated graphite is a well-suited material for this preliminary computer study of TEM images in solids. Graphite intercalation compounds are quasitwo-dimensional materials, and the well-defined periodic structure in the $c$-direction makes it easier to measure the amount and nature of the disorder. Another useful feature of fluorine-GIC's for this study is the fact that the amount of disorder can be easily varied by changing the fluorine intercalate concentration. Lastly, we can correlate the computer results with numerous transport data that exhibit distinct disorder effects. ${ }^{7-9}$

Section II describes the process of digitizing and analyzing the TEM pictures. In Sec. III, the technique is applied more specifically to fluorine-intercalated graphite fibers, and specific results on the microstructure of this material are obtained. In Sec. IV, a model is proposed that describes the origin of the disorder in fluorine GIC's.

\section{IMAGE ANALYSIS SYSTEM}

We have used the image analysis system TOSPIX-i (Toshiba Co. Ltd.) to carry out an image analysis of TEM pictures. The TOSPIX-i system consists of a workstation and the hardware for image analysis, an ITV (Industrial Television) camera, and a copy machine.

In the operation of the image analyzer system, the TEM image is first transferred to an array of $512 \times 512$ Charge Coupled Devices (CCD's) in the ITV camera that records an analog version of the TEM picture. The original TEM image [Fig. 1(a) for the $\mathrm{C}_{9.0} \mathrm{~F}$ image and Fig. 1(b) for the $\mathrm{C}_{4.5} \mathrm{~F}$ image] and the analog images obtained from the CCD recordings are shown in Figs. 1 and 2, respectively, for fluorine-intercalated graphite fibers with stoichiometries $\mathrm{C}_{9.0} \mathrm{~F}$ and $\mathrm{C}_{4.5} \mathrm{~F}$ [Figs. 2(c) and $2(\mathrm{e})]$, and for a pristine graphite fiber heat-treated at $2950{ }^{\circ} \mathrm{C}$ [Fig. 2(a)]. The typical size of a picture corresponds to an area of $220 \AA$ by $220 \AA$, so that each picture contains information on many atoms. The exact size of the recorded area depends, of course, on the resolution of the TEM image. The optimum picture size is determined
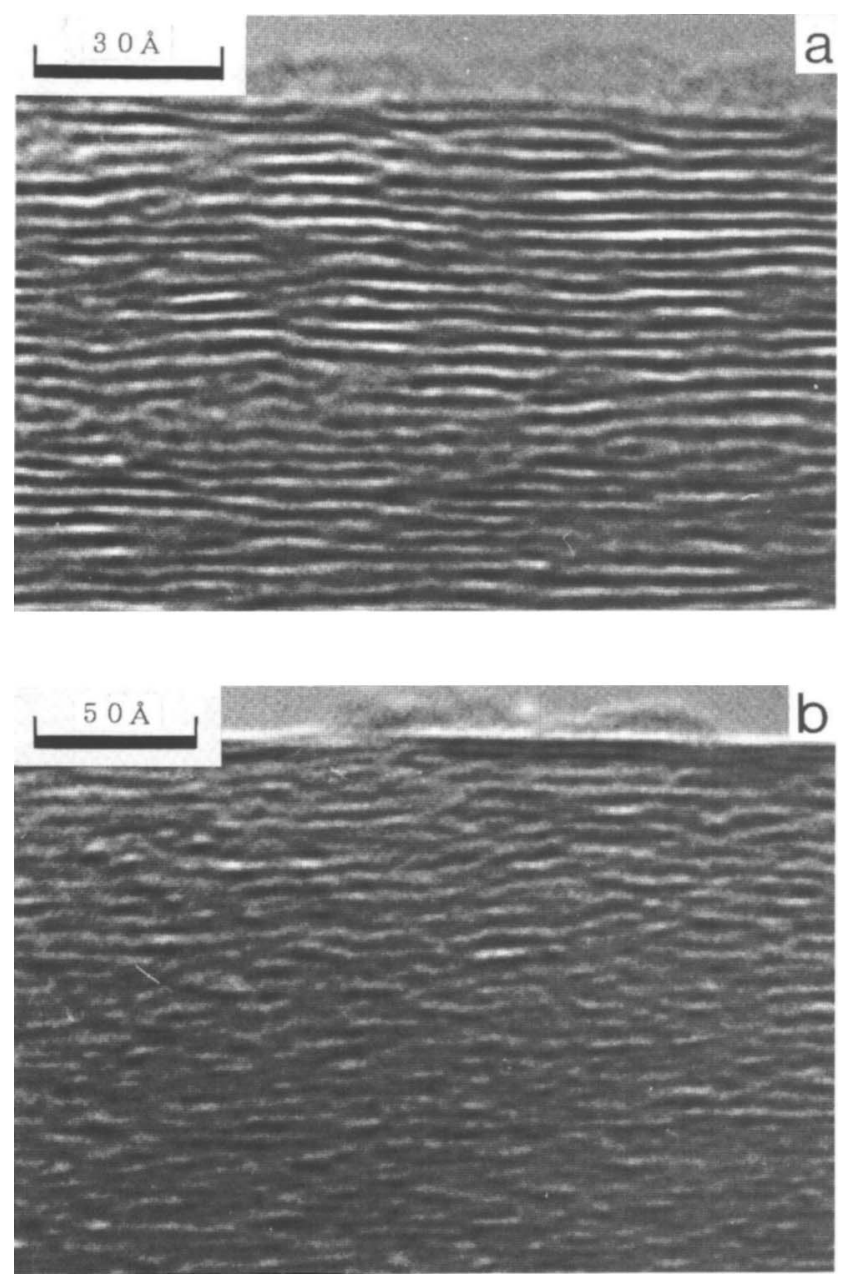

FIG. 1. (a) The Transmission Electron Microscopy (TEM) image of fluorine-intercalated graphite fibers with stoichiometry $\mathrm{C}_{9.0} \mathrm{~F}$. (b) The Transmission Electron Microscopy (TEM) image of fluorineintercalated graphite fibers with stoichiometry $\mathrm{C}_{4.5} \mathrm{~F}$.

by a trade-off between getting as many atoms as possible into the picture, in order to obtain a good statistical average of the density and type of defects present in the sample, and having a high enough resolution so as to be able to obtain a precise estimate of the interlayer spacing or the in-plane domain size. We estimate the resolution of the original TEM picture combined with the present image analysis to be about $0.1 \AA$, and the TEM pictures were taken under an acceleration voltage of $300 \mathrm{kV}$ and $400 \mathrm{kV}$ which have a resolution power of the lattice of 1.4 $\AA$. TEM micrographs were obtained at the focus position that gave the most clear contrast of the lattice fringes, for example about $65-80 \mathrm{~nm}$ under-focus using an acceleration voltage of $300 \mathrm{kV}$. Such TEM conditions have a broad and flat peak for the contrast transfer function, which allow formation of contrast in the lattice fringes within a range of 2.5-10 $\AA$. The TEM lattice spacings thus obtained have been very consis- 

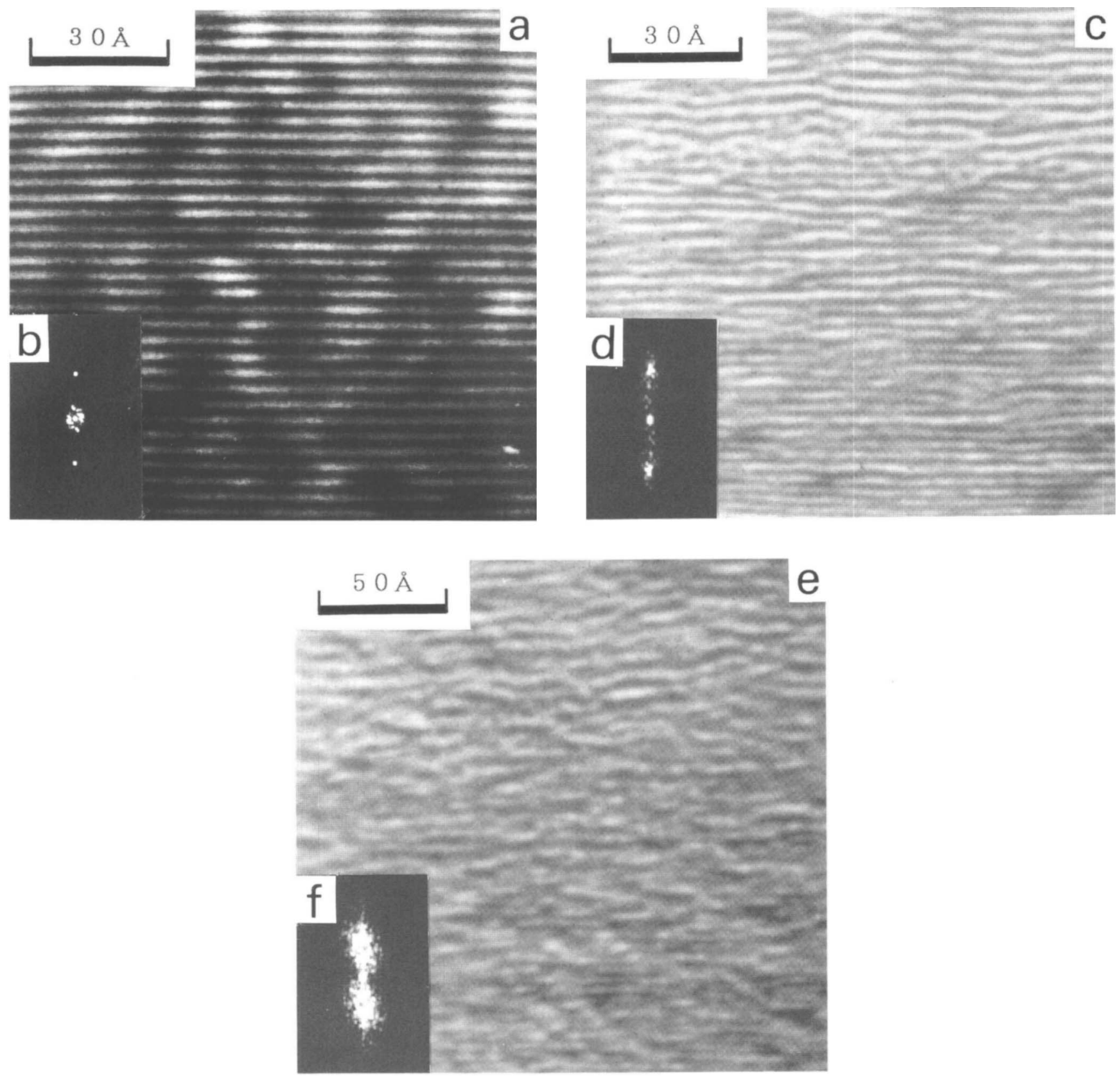

FIG. 2. The digitized pictures obtained from the CCD recording of a TEM picture corresponding to (a) pristine graphite fibers prior to fluorine intercalation, (c) fluorine intercalated graphite fibers with stoichiometry $\mathrm{C}_{9.0} \mathrm{~F}$, and (e) fluorine intercalated graphite fibers with stoichiometry $\mathrm{C}_{4.5} \mathrm{~F}$. The insets (b), (d), and (f) are the fast Fourier transforms (FFT) of (a), (c), and (e).

tent with the independently measured $\mathrm{x}$-ray diffraction results.

From the output of the CCD's, all pixels of the image are recorded on a brightness scale from 0 to 255. For this purpose, we first carry out a fast Fourier transform (FFT) of the analog picture shown in Figs. 2(b), 2(d), and 2(f). It is important to recognize that with the present digital method there is no astigmatism in the images from the FFT patterns. We then perform a high-pass filter operation, which keeps the high frequency components of the signal and discards the low frequency components [see, for example, Figs. 3(a), 4(a), and 5(a)]. The cut-off frequency corresponds to signals with a wavelength larger than 3 to 5 pixels, which corresponds approximately to 30 to $40 \AA$, although the exact cut-off frequency depends on the scale of the picture. Since these wavelengths are much larger than the average interlayer spacing, no physically interesting component of the signal is lost in this filtering operation. Quite the opposite, using signal processing, we are able to eliminate most of the 

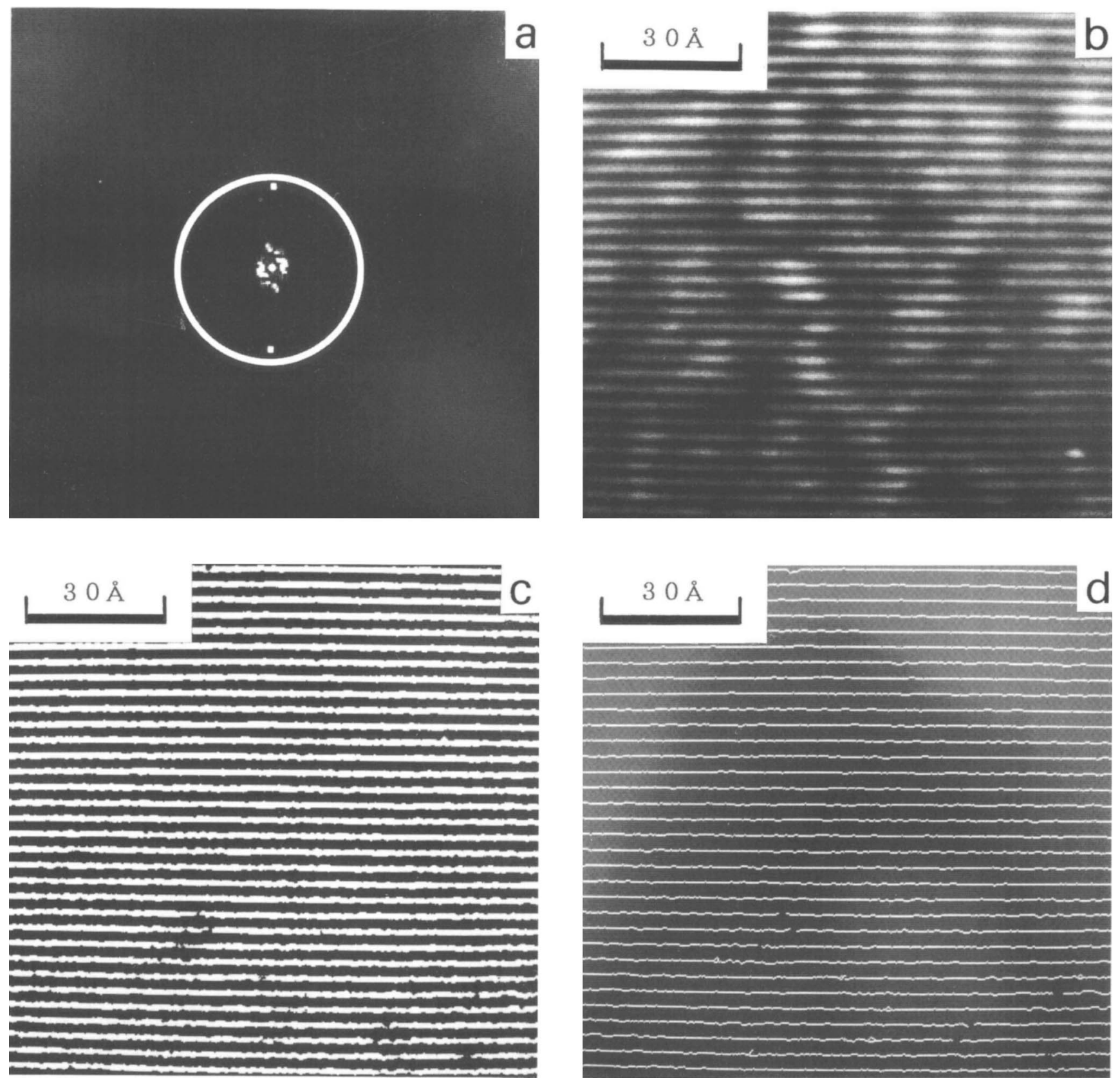

FIG. 3. (a) The mask applied to the power spectrum of Fig. 2(b) for the pristine fiber to eliminate the high and low frequency noise. (b) The image of the inverse fast Fourier transform (IFFT) after removing the high-frequency noise. The noise is removed by discarding the signal outside of the large circle in (a). (c) The binary image after removing the low-frequency noise. The noise is removed by discarding the signal inside the small central circle in (a). (d) A thinning process is applied to make the fringes of (c) appear more clearly defined.

noise, thereby focusing attention on the useful information contained in the picture [see Figs. 3(b), 4(b), and $5(b)]$. Similarly, if we are interested only in the wavelengths corresponding to unintercalated and intercalated graphite, we can eliminate most of the signal corresponding to repeat distances smaller than $2 \AA$ by discarding the high-frequency signal from outside the large outer circle [see Fig. 3(a)]. An inverse fast Fourier transform (IFFT) yields the real space picture after high and low frequency filtering [see Figs. 3(c), 4(c), and 5(c)].
It should be mentioned here that the reciprocal space diffractogram of the sample, instead of being obtained numerically from the FFT of the real-space TEM image, can be obtained optically from the TEM microscopy without any numerical operations. ${ }^{4}$ Indeed, the realspace image shown in Fig. 1 is really a double Fourier transform of the original picture. One might expect the TEM FFT analog image to be more accurate and less noisy than the same image obtained by means of numerical calculation. The drawback of the optical diffrac- 

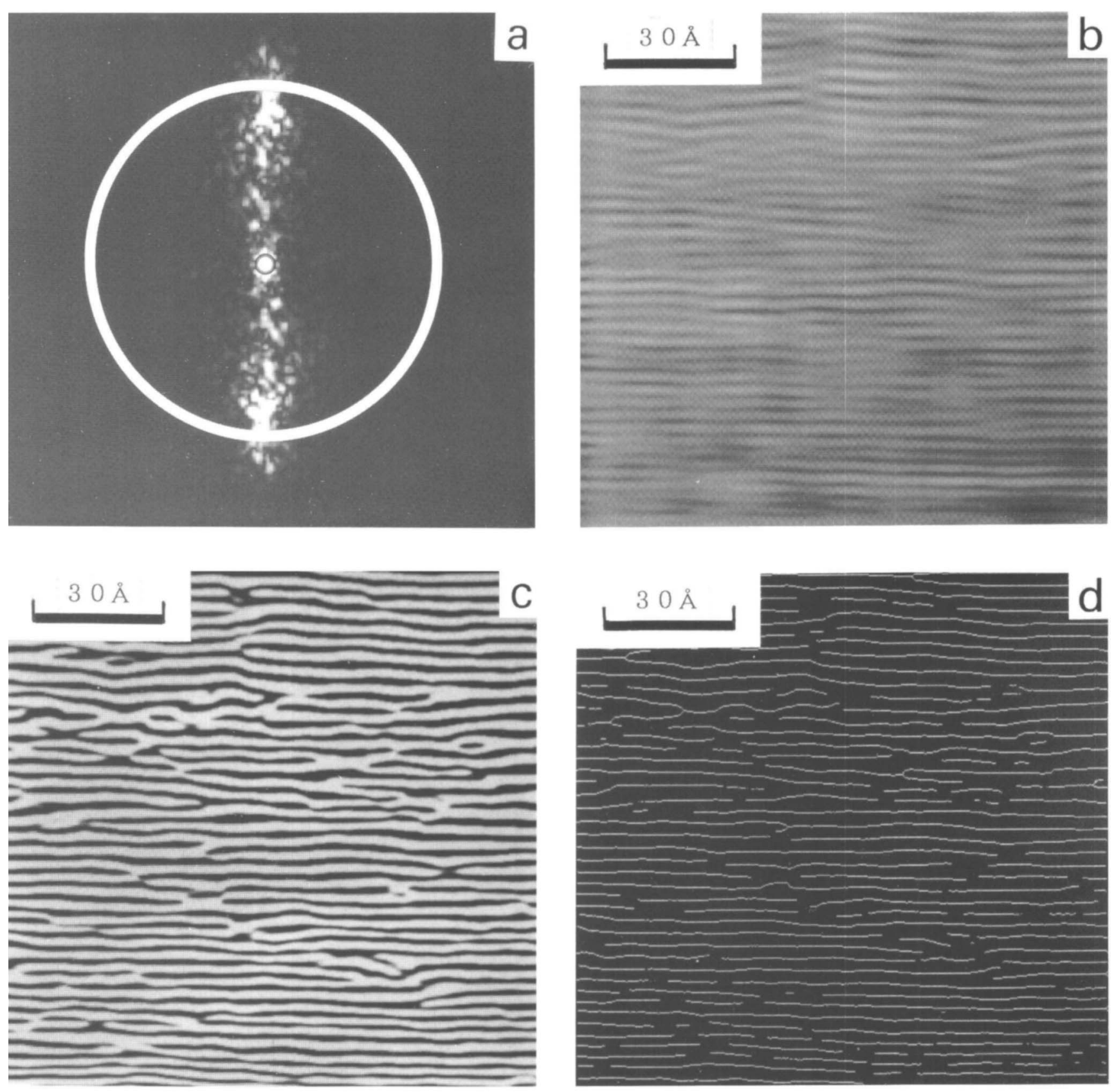

FIG. 4. The same operations as in Fig. 3 are applied to the picture of Fig. 2(d), i.e., to a $\mathrm{C}_{9.0} \mathrm{~F}$ fiber.

togram is that, because of the aberration present in the electron microscope, the signal present in the reciprocal space image comes from a region of the sample on the order of $1 \mu \mathrm{m}$, as opposed to approximately $200 \AA$ for the real space picture used in the present work.

In carrying out the digitization procedure, the analog picture obtained from the CCD array [e.g., Fig. 2(a)] is then digitized on a scale of 0 to 255 , i.e., on the basis that any signal brighter than 128 will yield a 1 and any signal less bright than 128 will yield a 0 , to form the binary image. It has been checked that the picture obtained, and especially the interlayer distances are not sensitive to the threshold value of 128 for discriminating between 0 and 1. Indeed, in the fluorine-based GIC's cut-off values of 100 or 140 did not yield a significantly different result.

The next step in analyzing the digitized picture is to carry out a FFT of the real-space picture, using the formula:

$$
\begin{aligned}
& F(u, v)=\frac{1}{M N} \sum_{m=-M / 2}^{M / 2-1} \sum_{n=-N / 2}^{N / 2-1} \\
& f(m, n) \exp \left[-2 i \pi\left(\frac{m u}{M}+\frac{n v}{N}\right)\right]
\end{aligned}
$$



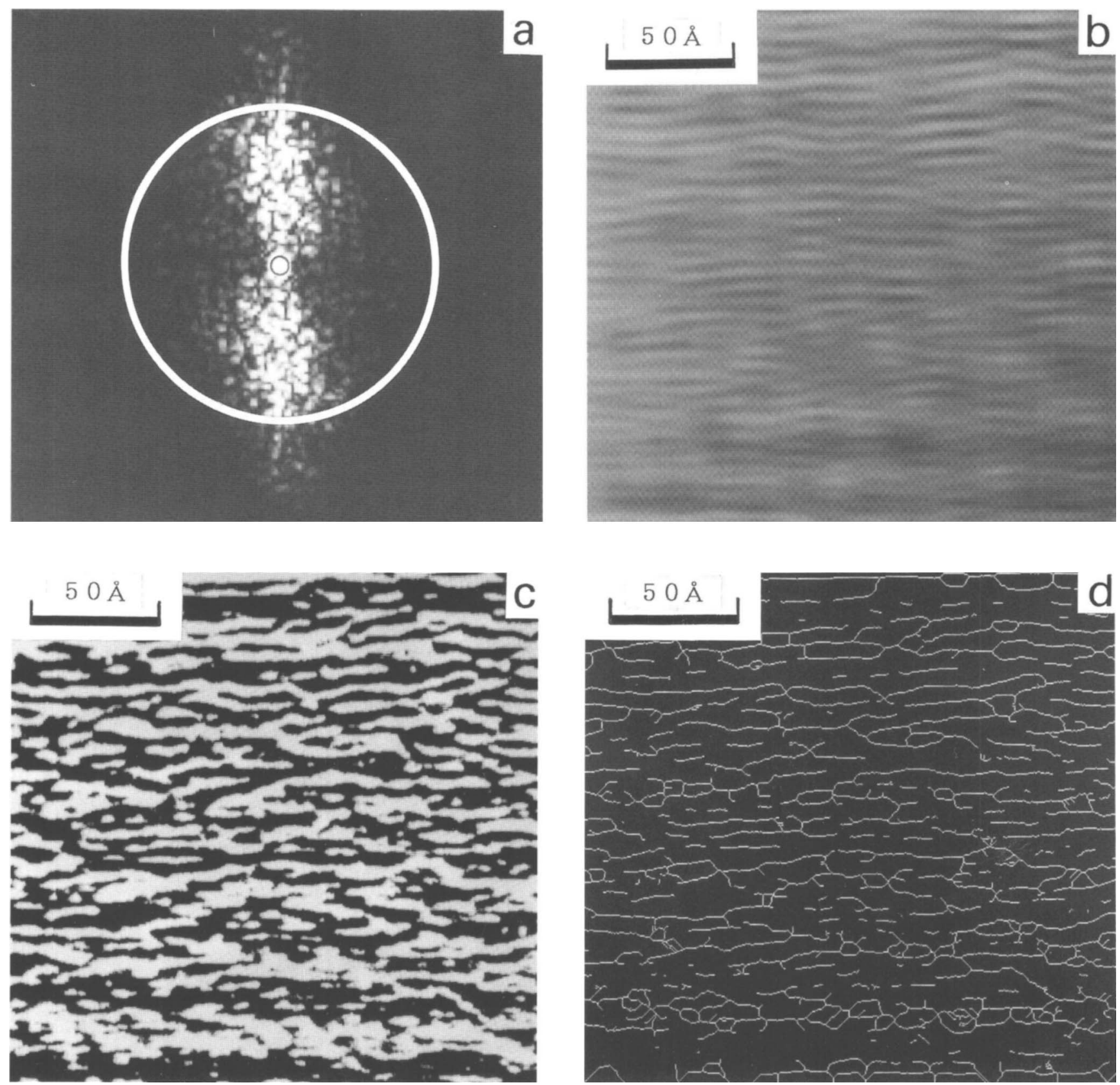

FIG. 5. The same operations as in Fig. 3 are applied to the picture of Fig. 2(f), i.e., to a $\mathrm{C}_{4.5} \mathrm{~F}$ fiber.

in which $M$ is the total number of pixels along the $x$-axis, $m$ is the corresponding pixel index, and $u$ is the frequency corresponding to $x$-axis periodicities. $N, n$, and $v$ are the corresponding symbols for the $y$-axis. If $f(m, n)$ denotes the information contained in the digitized pattern of the TEM pictures, then $F(u, v)$ denotes the corresponding diffractogram in reciprocal space, which can in turn be used to generate $f(m, n)$ using an IFFT. ${ }^{10}$

The formation of the lattice fringe TEM pattern involves an interference between the $(000)$ and $(00 l)$ beams in the electron microscope. In this sense, the lattice fringe pattern is not a direct projection of the real lattice. In particular, we do not claim that the black lines represent graphene or fluorine layers and the white areas represent vacuum or vice versa. However, we do believe that the periodicities of the physical lattice and the relative intensities of these periodicities are preserved. Since we are interested only in the contrast between the white and black signals, i.e., interlayer spacings and in-plane coherence lengths, exact knowledge of the physical meaning of the signal of the TEM picture is not necessary. 


\section{APPLICATION TO FLUORINE-INTERCALATED GRAPHITE FIBERS}

The digitized pictures of two fluorine-intercalated graphite fibers with concentrations $\mathrm{C}_{9.0} \mathrm{~F}$ and $\mathrm{C}_{4.5} \mathrm{~F}$ are shown in Figs. 4 and 5, respectively. To test our method of analysis of TEM pictures, we have analyzed a TEM picture for a pristine fiber prior to fluorine intercalation in the same way as was done for the $\mathrm{C}_{x} \mathrm{~F}$ fibers, and the results are shown in Fig. 3. It is evident that the host fiber has a high degree of lattice perfection, the lattice fringe pattern consisting of very long and straight fringes. The pristine material before intercalation was a vapor-deposited graphite fiber, with a diameter of $\approx 0.1-2.0 \mu \mathrm{m}$, heat-treated in an argon atmosphere to a temperature $T_{H T}=2950{ }^{\circ} \mathrm{C}$. The process of intercalating these fibers with fluorine has been described elsewhere. ${ }^{11,12}$ These fibers are stage-II and stage-I fluorine GIC's, respectively; i.e., there are two or one graphene planes sandwiched between sequential layers of fluorine. ${ }^{12}$ It is experimentally observed that an increase in the concentration of the intercalated fluorine species corresponds to an increase in the amount of disorder, consistent with our model that this disorder originates from the intercalation process. That this is so is proved by the very ordered nature of the pristine fibers, as measured by a number of techniques. ${ }^{13}$ From the binary image and the thinning process, the graphite lattice fringes are essentially perfectly depicted in Fig. 3(d).

As described in the previous section, we carry out a FFT of the TEM image to obtain an image of the fiber in reciprocal space, as shown in Figs. 3(a), 4(a), and 5(a). If the fibers were perfectly ordered, we would expect to obtain sharp spots along the $y$-axis, corresponding to the interlayer repeat distance and its harmonics. (The $y$-axis is taken as the axis perpendicular to the graphene layers.) If the intensity of the lattice fringes has a cosinusoidal dependence in the $y$-direction, then only the fundamental periodicity would appear, whereas a square wave dependence of the intensity would emphasize the contributions of the harmonics in the FFT pattern. The harmonic content found in the lattice fringe images for the highly ordered vapor grown fibers prior to intercalation is shown in Fig. 2(b) where the FFT pattern is presented, while Fig. 6 shows the distribution in the real space periodicities obtained from the FFT of Fig. 3(a).

The size of the diffuse spots provides a sensitive measure of the disorder. The extension of the spots in Figs. 4 and 5 along the $x$-axis measures the presence of disorder in the planes, whereas the extension along the $y$-axis corresponds to a spread in the distribution of interlayer spacings. The distribution of interlayer spacings corresponds to a variation of the fluorine intercalate concentration throughout the sample, which is thus

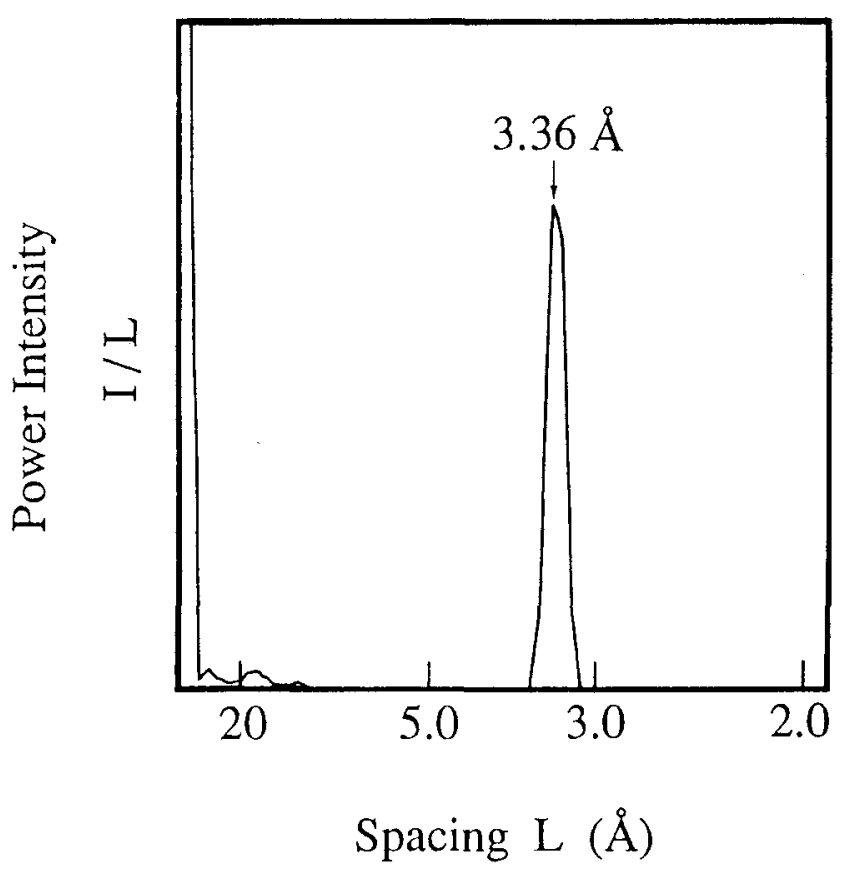

FIG. 6. The distribution of interlayer repeat distances for the pristine fiber obtained from the FFT image of Fig. 3(a).

seen to be inhomogeneous. As expected, the spread of the spot intensities in the reciprocal space diffractogram is more important for the fiber with the higher fluorine concentration $\mathrm{C}_{4.5} \mathrm{~F}$. We obtain typical in-plane coherence lengths of $11.5 \AA$ and $6.8 \AA$ for the $\mathrm{C}_{9.0} \mathrm{~F}$ and $\mathrm{C}_{4.5} \mathrm{~F}$ fibers, respectively. Since the in-plane coherence lengths of the graphene planes in $\mathrm{C}_{x} \mathrm{~F}$ fibers, obtained by Raman scattering measurements, are much higher, on the order of $40 \AA$ for a $\mathrm{C}_{4.5} \mathrm{~F}$ fiber, ${ }^{14}$ we argue that the very short coherence lengths that are observed in the TEM pictures are associated with the random structure of the fluorine intercalate. The very small inplane coherence distances that are observed indicate that the fluorine is not in registry with the carbon layers, allowing a range of local stoichiometries. Because of the dependence of the bonding and $c$-axis repeat distance on fluorine concentration, ${ }^{15}$ the observed $I_{c}$ values can vary from one carbon site to another in the basal plane.

From the reciprocal space diffractogram, it is possible to obtain the distribution of the interlayer spacings in the pristine as well as in the fluorine-intercalated carbon fibers. This is done by integrating the FFT of the pictures in Figs. 3(a), 4(a), and 5(a) along the $y$-axis, in a small strip of thickness one pixel along the $x$-axis. One pixel along the $y$-axis corresponds approximately to a thickness $0.15 \AA$ in real space for frequencies around the $c$-axis repeat distance in reciprocal space. The distribution of interlayer repeat distances $L(\AA)$ is shown in Figs. 6, 7, and 8 for a pristine fiber and for the $\mathrm{C}_{9.0} \mathrm{~F}$ and $\mathrm{C}_{4.5} \mathrm{~F}$ fibers, respectively. The spacing $L$ $(\AA)$ in the figures nearly corresponds to the continuous 


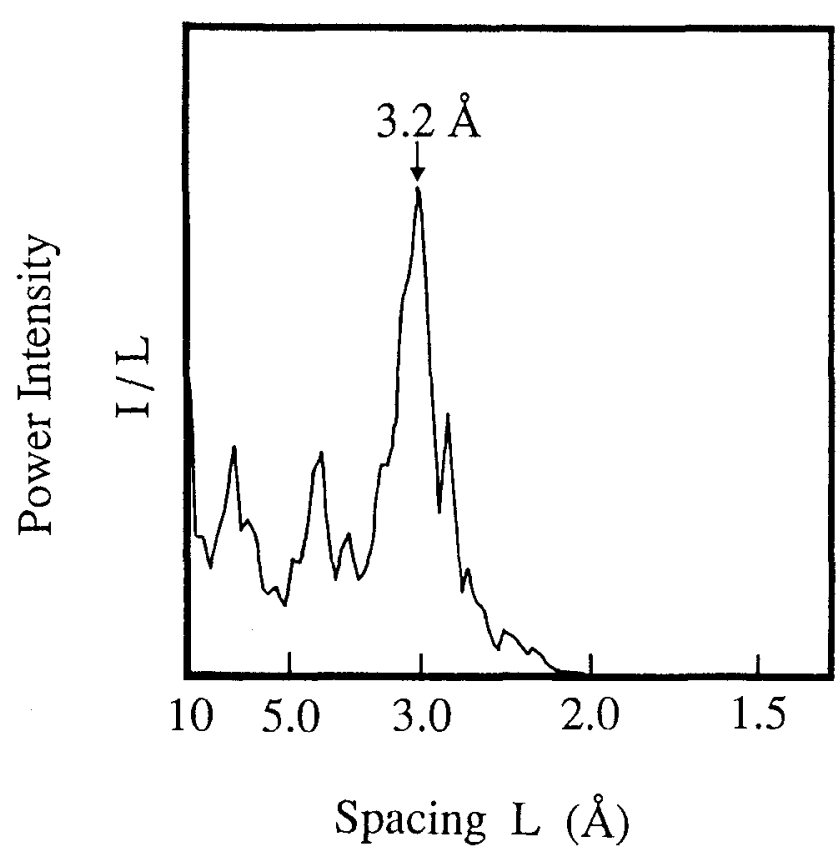

FIG. 7. The distribution of interlayer repeat distances for the $\mathrm{C}_{9.0} \mathrm{~F}$ fiber obtained from the FFT image of Fig. 4(a).

region of the microscopic transfer function which gives enough image contrast to form the fringes.

A very sharp peak is clearly demonstrated for the host fiber in Fig. 6 . In Fig. 7 for the $\mathrm{C}_{9.0} \mathrm{~F}$ fiber, we can clearly observe one large peak around $3.2 \AA$. This value is consistent with $\mathrm{x}$-ray diffraction results showing a sharp (002) peak for pristine fibers $\left(d_{002}=3.365 \AA\right)$ and a broad $(003)$ peak for $\mathrm{C}_{9.0} \mathrm{~F}$ fibers $\left(d_{003}=3.149 \AA\right)$, respectively. The interlayer

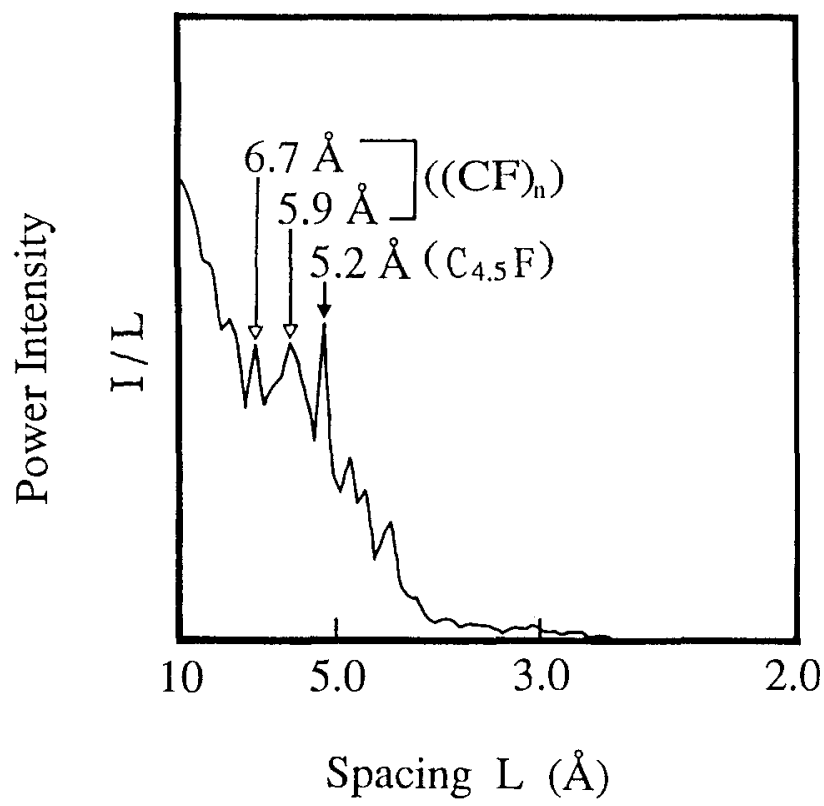

FIG. 8. The distribution of interlayer repeat distances for the $\mathrm{C}_{4.5} \mathrm{~F}$ fiber obtained from the FFT image of Fig. 5(a). value of $3.2 \AA$ roughly corresponds to the usual distance between graphite planes in pristine graphite $(3.36 \AA)$, so that some of the line intensity may have a contribution from unintercalated regions of the $\mathrm{C}_{9.0} \mathrm{~F}$ fiber. Another possible explanation for the sharp peak at $3.2 \AA$ may be due to a $d_{003}$ diffraction contribution from stage II regions of the sample. Because of the broadness of the peak at $3.2 \AA$, it is difficult to differentiate between the intercalated and unintercalated regions of the $\mathrm{C}_{9.0} \mathrm{~F}$ fiber using this technique. Also present in Fig. 7 is a more diffuse peak corresponding to an interlayer distance of $\approx 4.6 \AA$, perhaps indicating the presence of a small amount of stage-I material in this predominately stage-II sample.

As for the more concentrated $\mathrm{C}_{4.5} \mathrm{~F}$ sample (see Fig. 8), there is no sign of the presence of regions with interlayer separation of $3.36 \AA$, which is the interlayer separation for single crystal graphite before intercalation. A peak with repeat distance $5.2 \AA$ corresponding to the stage-I material can clearly be observed. (The $c$-axis repeat distance as obtained from $\mathrm{x}$-ray diffraction for this sample is $5.16 \AA$.) Several other peaks are also present that correspond either to more dilute regions or to some noise arising from the disorder present in these fibers.

The chemical bonding of fluorine in graphite is quite special, in that fluorine can form both ionic and covalent bonds with carbon. Numerous experiments have demonstrated this dual ionic and covalent behavior of fluorine in graphite. ${ }^{15-19}$ We expect the interlayer repeat distance to change slightly, depending on whether we are looking at a covalent or an ionic bond between fluorine and carbon, with the covalent bond being slightly longer than the ionic bond. ${ }^{15}$ Unfortunately, the resolution of the FFT diffractogram is not good enough to enable us to clearly resolve the fluorine peak into two peaks corresponding to covalent and ionic bonding. As seen from Fig. 8, it is suggested that the more concentrated $\mathrm{C}_{4.5} \mathrm{~F}$ sample has greater disorder, which induces a larger spread in interlayer repeat distances, presumably corresponding to repeat distances of the covalent compound $(\mathrm{CF})_{n} \cdot{ }^{18}$

With digital signals, it is possible to select a specific peak by using filters and discarding any signal corresponding to other interlayer repeat distances. Then, carrying out the inverse fast Fourier transform of the filtered reciprocal space diffractogram yields the distribution in real space of regions with the selected interlayer spacing. This digital process has a large intrinsic advantage over the conventional optical interferogram approach because of the easy inverse transformation to a real space image that can be made digitally. We have carried out this operation for the unintercalated and intercalated graphite peaks, respectively. The result of this operation is shown in Fig. 9 for a $\mathrm{C}_{9.0} \mathrm{~F}$. The typical island size in Fig. 9 is found to be on the order of approximately $70 \AA$ and is identified with the unintercalated regions. 

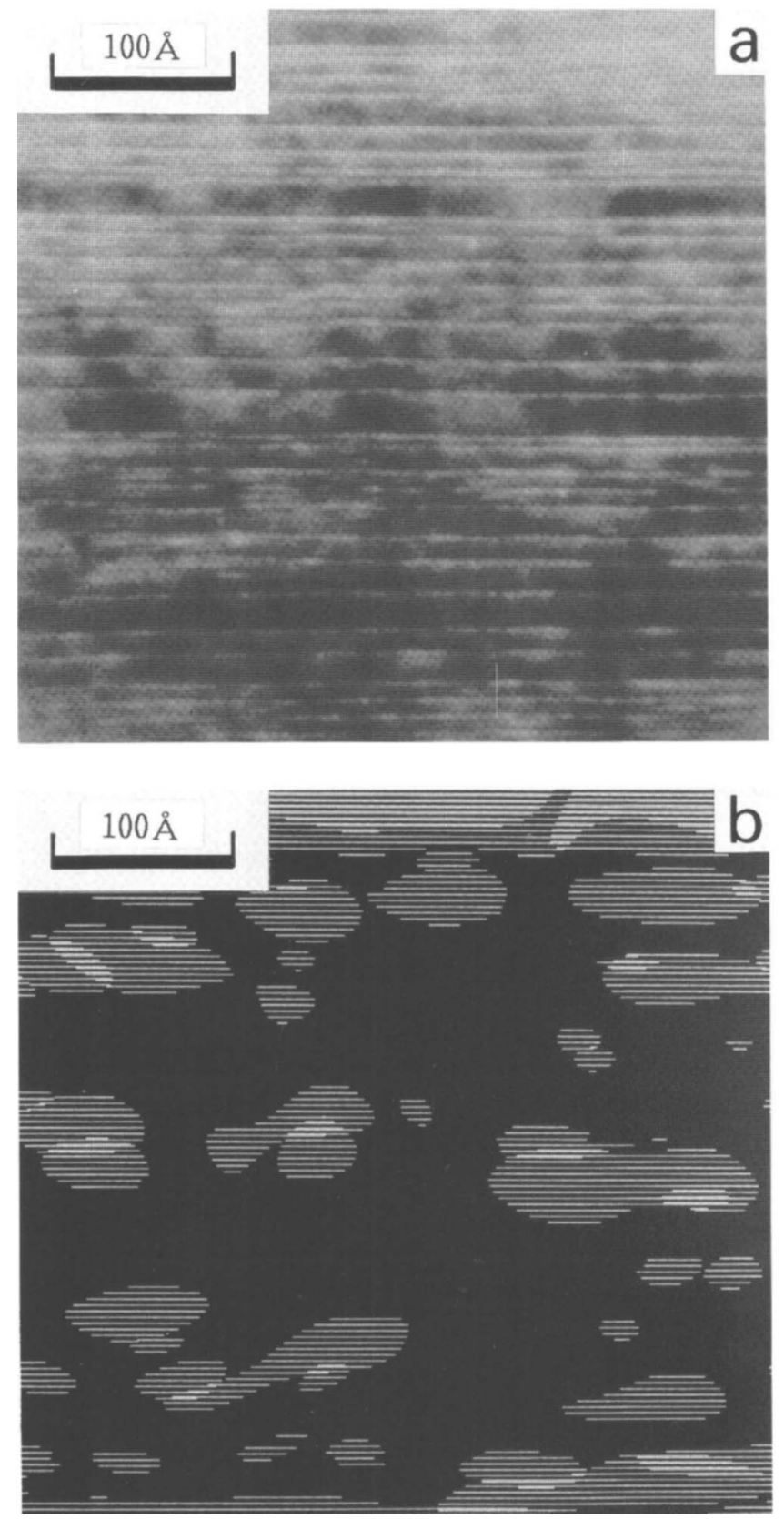

FIG. 9. (a) The digitized picture determined by the CCD recording of the TEM picture, which was obtained from a different $\mathrm{C}_{9.0} \mathrm{~F}$ fiber. (b) The regions of the material corresponding to unintercalated graphite, i.e, to an interlayer distance $3.3 \pm 0.1 \AA$. A characteristic island structure is obtained.

On the other hand, Fig. 10 shows three sets of pictures for $\mathrm{C}_{4.5} \mathrm{~F}$ fibers corresponding to the regions with interlayer repeat distances $5.1 \AA, 5.4 \AA$, and $5.9 \AA$, respectively. X-ray diffraction gives a broad peak with an average $I_{c}$ value of $5.16 \AA$ for this sample. The high frequency components are not included for the IFFT. The resulting images exhibit rather straight fringes. It is obvious from Fig. 10 that regions with the same interlayer spacing form an island structure.

\section{ANALYSIS OF THE TEM RESULTS}

It was already established through numerous transport and optical experiments that the properties and structure of fluorine GIC's are quite different from that of most other known GIC's with regard to the dual nature of the C-F bond (ionic and covalent), and with regard to variation in $I_{c}$ value with fluorine concentration. . $^{12,15-17,19}$ In the light of the TEM results described above, we propose a model for the microstructure of fluorine GIC's.

A material with nominal composition $\mathrm{C}_{x} \mathrm{~F}$ is found to consist of islands within which the compositional variation is small, but the sample as a whole has a large spread in fluorine concentrations. The island behavior is consistent with the observation that it is possible to make compounds of the same nominal stage, but with a wide range of fluorine concentrations. It is found experimentally possible to further increase the concentration of intercalated fluorine after stage I has already been reached, as opposed to commensurate GIC's such as K-GIC's where stage I corresponds to the $\mathrm{C}_{8} \mathrm{~K}$ stoichiometry, and more concentrated samples $\left(\mathrm{C}_{6} \mathrm{~K}, \mathrm{C}_{3} \mathrm{~K}\right)$ cannot generally be fabricated unless pressure is used. ${ }^{20}$

As the fluorine concentration increases, the sample remains inhomogeneous, but the average fluorine concentration of the islands increases. This behavior is probably related to the dual covalent and ionic nature of the chemical bond between carbon and fluorine, since the bond length depends on the nature of the bond. For the covalent bond, the charge remains localized between the $\mathrm{C}$ and the $\mathrm{F}$, thereby lengthening the bond. For the ionic bond, the charge is transferred to the graphene layers, thereby resulting in a different charge distribution in the interlayer region between graphene sheets.

In this connection, we offer the following explanation for the formation of islands in the $\mathrm{C}_{x} \mathrm{~F}$ material. The strain energy in $\mathrm{C}_{x} \mathrm{~F}$ is high for a fluorine distribution where neighboring interlayer distances are different, since this would introduce a large amount of waviness in the graphene layers throughout the sample. The strain energy is correspondingly reduced by the formation of islands of material with the same interplanar separation within an island, so that an increased average fluorine concentration is accommodated by the growth of fluorine-rich regions with a greater interlayer separation at the expense of fluorine-poor regions. In this model, the strain energy is concentrated at the boundary between islands with different fluorine concentrations. The island size is determined by the relative magnitudes of the strain energies within the bulk of the island and the free energy of the contact surface between islands.

TEM lattice image pictures show very characteristic behavior from one intercalate species to another. For example, $\mathrm{CuCl}_{2}-\mathrm{GIC}^{2}{ }^{21}$ show very straight fringes, while K-GIC's and KH-GIC's show more wavy lattice 

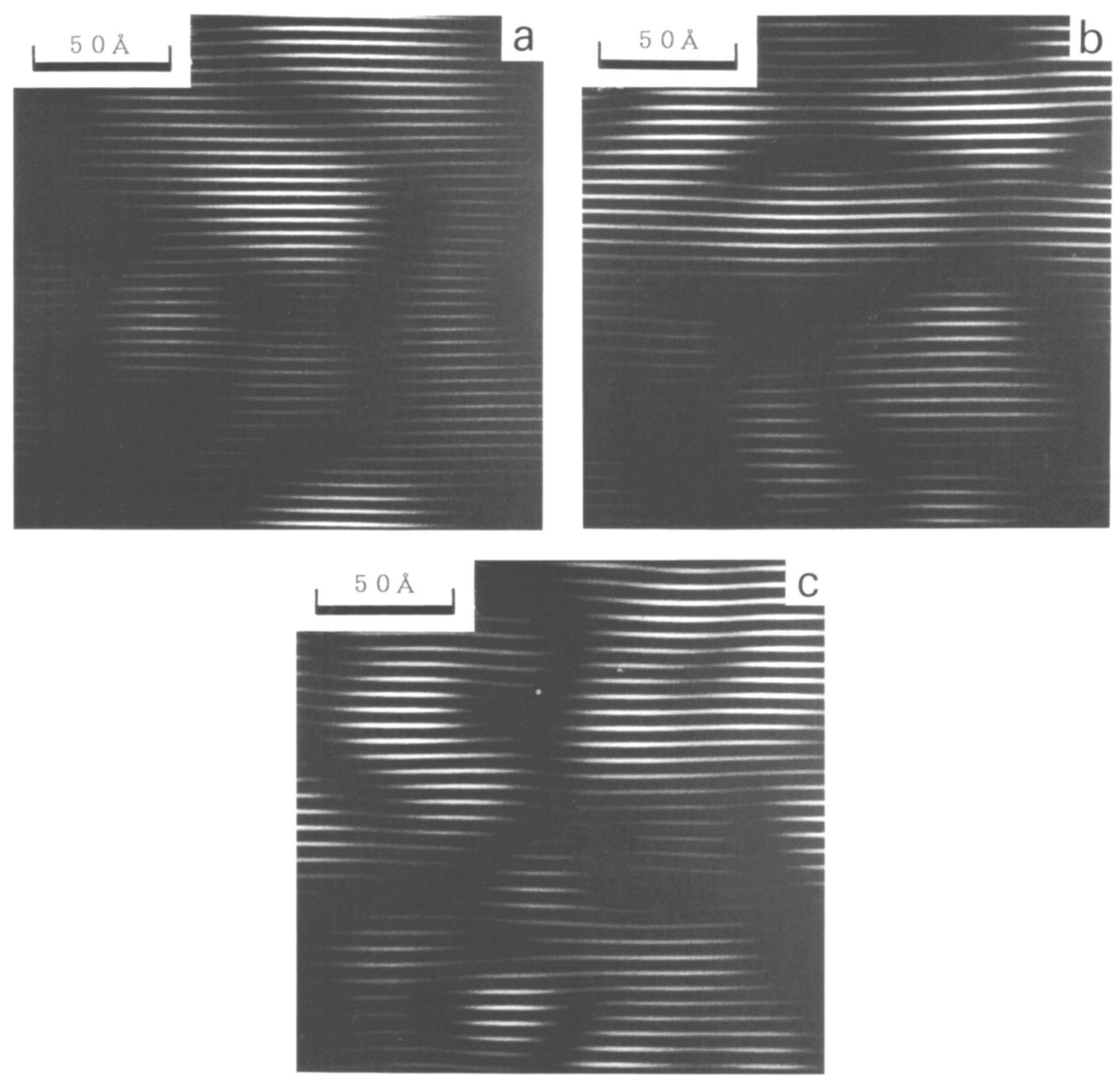

FIG. 10. Typical island structures for the regions of the sample with different interlayer spacings of (a) $5.1 \pm 0.1 \AA$, (b) $5.4 \pm 0.1 \AA$, and (c) $5.9 \pm 0.1 \AA$, respectively, which are obtained from the IFFT of a stage-I $\mathrm{C}_{4.5} \mathrm{~F}$ sample.

image patterns, reflecting a higher degree of intercalateinduced disorder. Furthermore, these features of the lattice structure are reflected in the transport properties measured on these GIC's. Because of the special features of F-GIC's relative to other graphite intercalation compounds (discussed above), the $\mathrm{C}_{x} \mathrm{~F}$ fibers show very wavy and disordered lattice fringe patterns.

Within the framework of this model, we can also account for the scattering of carriers measured in transport experiments. ${ }^{7-9,11,19}$ The mean free path $\lambda$ of holes in F-GIC's increases from $\approx 5 \AA$ in $\mathrm{C}_{3.6} \mathrm{~F}$ to many tens of $\AA$ in a dilute compound such as $\mathrm{C}_{5.8} \mathrm{~F}$ or $\mathrm{C}_{6.6} \mathrm{~F} . \lambda$ is thus much smaller than the typical size of the islands present in fluorine GIC fibers, and we attribute scattering events to local changes in the bonding between fluorine and carbon atoms. Because ionic bonds are shorter than covalent bonds, the proximity of ionically and covalently bonded carbon atoms disturbs the graphene layers by pushing the covalently bonded graphene atoms to out-of-plane positions, thus scattering the conduction holes. As mentioned above, as the fluorine intercalate concentration increases, the proportion of covalent bonds 
increases, which accounts for the rapid decrease in the hole mean free path.

\section{CONCLUSION}

We have carried out a computer analysis of TEM pictures of fluorine-intercalated graphite fibers as well as their host graphite fibers. In so doing, we have shown that a computer treatment of digitized images can provide a very useful tool to reduce the level of noise and to obtain quantitative estimates for the various structural parameters. Using this analysis, we propose a model for the defect structure of fluorineintercalated graphite fibers that accounts for both the structural defects observed with TEM microscopy and for the disorder effects measured using transport experiments. Although $\mathrm{C}_{x} \mathrm{~F}$ fibers are a disordered material if compared to most other GIC's, the $\mathrm{C}_{x} \mathrm{~F}$ fibers retain a large amount of order, as is evident from the TEM studies and from Raman scattering results. We plan in the future to use the present technique of computer analysis of TEM images to characterize even more disordered carbon-based solids.

\section{ACKNOWLEDGMENTS}

This work was supported by NSF grant DMR8819896, and a travel grant from the US-Japan Cooperative Research Program NSF INT-8714964.

\section{REFERENCES}

1. M. S. Dresselhaus, G. Dresselhaus, K. Sugihara, I. L. Spain, and H. A. Goldberg, in Graphite Fibers and Filaments (SpringerVerlag, Berlin, 1988), Vol. 5 of Springer Series in Materials Science.

2. M. S. Dresselhaus, H. A. Goldberg, and I. L. Spain, in Proceedings of the 1985 CRDC Scientific Conference on Obscuration and Aerosol Research, edited by R. H. Kohl, Vols. I and II, 291-324 (1985).

3. J. C.H. Spence, in Experimental High Resolution Electron Spectroscopy (Clarendon Press, Oxford, 1981).
4. P. Hirsch, A. Howie, R. B. Nicholson, D. W. Pashley, and M. J. Whelan, in Electron Microscopy of Thin Crystals (Robert E. Krieger Publishing Co., New York, 1977).

5. A. Howie, in Theory of Electron Diffraction Image Contrast in Electron Microscopy of Materials Science, edited by U. Valdre and A. Zichichi (Academic Press, New York, 1971).

6. M.S. Dresselhaus and J.S. Speck, in Intercalation in Layered Materials, edited by M.S. Dresselhaus (Plenum Press, New York, 1987), p. 213.

7. S. L. di Vittorio, M.S. Dresselhaus, M. Endo, and T. Nakajima, Phys. Rev. B 43, 12304 (1991).

8. S. L. di Vittorio, M.S. Dresselhaus, and G. Dresselhaus, in New Horizons in Low Dimensional Electron Systems-A Festschrift in honour of Professor H. Kamimura, edited by H. Aoki, M. Tsukada, M. Schlüter, and F. Lévy (Kluwer Academic Publishers, Dordrecht, 1991), p. 3.

9. S. L. di Vittorio, M. S. Dresselhaus, M. Endo, and T. Nakajima, Phys. Rev. B 43, 1313 (1991).

10. A. Rosenfeld and A. C. Kak, Digital Picture Processing (1976).

11. L. Piraux, J. Mater. Res. 5, 1285 (1990).

12. T. Nakajima, N. Watanabe, I. Kameda, and M. Endo, Carbon 24, 343 (1986).

13. M. S. Dresselhaus and G. Dresselhaus, Adv. Phys. 30, 139 (1981).

14. A.M. Rao, A.W.P. Fung, M.S. Dresselhaus, G. Dresselhaus, M. Endo, and T. Nakajima, Phys. Rev. B 45, 6883 (1992).

15. T. Nakajima, M. Molinier, and M. Motoyama, Carbon 29, 429 (1991).

16. I. Ohana, I. Palchan, Y. Yacoby, D. Davidov, and H. Selig, Phys. Rev. B 38, 12627 (1988).

17. T. M. Mallouk, B. L. Hawkins, M.P. Conrad, K. Zilm, G.E. Maciel, and N. Bartlett, Philos. Trans. R. Soc. London A314, 179 (1985).

18. N. Watanabe, T. Nakajima, and H. Touhara, in Graphite Fluorides (Elsevier, Amsterdam, 1988), Studies in Inorganic Chemistry, vol. 8.

19. S. L. di Vittorio, M. S. Dresselhaus, V. Bayot, L. Piroux, J.P. Issi, M. Endo, and T. Nakajima, in Defects in Materials, edited by P. D. Bristowe, J. E. Epperson, J. E. Griffith, and Z. LilientalWeber (Mater. Res. Soc. Symp. Proc. 209, Pittsburgh, PA, 1991), p. 329.

20. I.T. Belash, A.D. Bronnikov, O.V. Zharikov, and A.V. Pal'nichenko, Synth. Metals 36, 283 (1990).

21. M. Endo, T.C. Chieu, G. Timp, M.S. Dresselhaus, and B.S. Elman, Phys. Rev. B 28, 6982 (1983). 